\title{
ESTUDIO QUÍMICO DEL ACEITE ESENCIAL DE LA "YARETA" Smallanthus parviceps (Blake) Rob
}

\author{
Gianella Neves Ordóñez ${ }^{1}$, Marisol Fernández Ruiz ${ }^{1}$, Sugia Solís Miranda ${ }^{1}$, \\ Nino Castro Mandujano ${ }^{1}$, Carlos Serrano Flores ${ }^{1}$
}

\begin{abstract}
RESUMEN
En la presente investigación se realizó un estudio fitoquímico al aceite esencial de la "yareta" (Smallanthus Parviceps (Blake) Rob), recolectada en Ayacucho, en el distrito de Chumpi. Se obtuvo el aceite esencial por el método de destilación por arrastre con vapor de agua, a partir de $1,50 \mathrm{~kg}$ de planta fresca, su olor se puede definir como cítrico, agradable y balsámico, además el aceite esencial tiene un poder antiinflamatorio según la información etnobotánica de la planta. En cuanto a su caracterización, primero se determinó las propiedades fisicoquímicas, el cual nos indica que el aceite de "yareta" contiene $0,06 \%$ de aceite esencial cuya densidad es $0,75 \mathrm{~g} / \mathrm{mL}$ y de un $\mathrm{pH}: 6$, luego se determinó sus componentes por cromatografía gaseosa CGMS. Sus componentes mayoritarios son: $\alpha$-felandreno, p-cimeno, cariofileno, los isómeros $\alpha$-curcumeno y $\beta$-curcumeno, $\alpha$-pineno y oxido de cariofileno.
\end{abstract}

Palabras clave: yareta, alfa-felandreno, p-cimeno, cariofileno, aceite esencial.

\section{STUDY OF THE ESSENTIAL OIL OF THE "YARETA" Smallanthus parviceps (Blake) Rob.}

\begin{abstract}
S
In the present investigation, a phytochemical study was carried out on the essential oil of the "yareta" (Smallanthus parviceps (Blake) Rob), collected in Ayacucho in the Chumpi district. The essential oil was obtained by the distillation method by dragging with steam, from 1.50 $\mathrm{kg}$ of fresh plant, its smell can be defined as citric, pleasant and balsamic, in addition the essential oil has an anti-inflammatory power according to the ethnobotanical information of the plant. Regarding its characterization, the physicochemical properties were first determined, which indicates that "yareta" oil contains $0.06 \%$ of essential oil whose density is $0,75 \mathrm{~g} / \mathrm{mL}$ and a $\mathrm{pH}: 6$, then its components were determined by CG-MS gas chromatography. Its major components are: $\alpha$-felandrene, $p$-cymene, caryophyllene, the $\alpha$-curcumene isomers and $\beta$-curcumene, to $\alpha$-pymene and to caryophyllene oxide.
\end{abstract}

Key words: yareta, alpha-felandrene, p-cymene, caryophyllene, essential oil.

${ }^{1}$ Facultad de Farmacia y Bioquímica. Universidad Nacional San Luis Gonzaga, Av. Los Maestros s/n Ica. felipesurco@gmail.com 


\section{INTRODUCCIÓN}

Los aceites esenciales son líquidos oleosos volátiles obtenidos de diferentes partes de plantas, son ampliamente usados en perfumería, cosmética, en la industria farmacéutica, agricultura, como aditivos en la industria alimentaria y remedios naturales, así como en aromaterapia. ${ }^{1}$ Las investigaciones previas han evidenciado que algunos aceites esenciales muestran algunas propiedades farmacológicas así como antiinflamatorias, antioxidante, antibacteriana, antimicótica, antimicrobiana ${ }^{2} \mathrm{y}$ otras actividades como insecticidas ${ }^{3}$.

El género Smallanthus fue descrito originalmente por Mackenzie en 1933, sobre la base de una sola especie norteamericana, Smallanthus uvedalius (L.) Mack. Posteriormente fueron transferidas a Smallanthus especies de otros géneros y descriptas nuevas, por diferentes autores. Actualmente, el género Smallanthus está constituido por 24 especies, distribuidas desde el sur-este de EEUU hasta el centro-norte de Argentina, con un mayor número de especies en Perú y México ${ }^{4}$.

Hay varias investigaciones del género Smallanthus, así, Guzmán 2011, realizó estudio fitoquímico del S. pyramidales $\mathrm{H}$ Bob, quienes realizaron estudios de aislamiento de metabolitos secundarios. 5 . Arnao, 2012, realizó una evaluación de la capacidad antioxidante de los extractos acuosos de la raíz y hojas del Smallanthus sonchifolius (yacón) ${ }^{6}$. Se realizó una búsqueda bibliográfica exhaustiva en diferentes bases de datos (ACS Publications, Science Finder, Scopus, Scielo) y no se ha encontrado estudios fitoquímicos de la especie en estudio. Las investigaciones encontradas giran con respecto a la familia Asteraceae, realizaron la extracción del aceite esencial del Smallanthus sonchifolius Poepp. \& Endl, mediante hidrodestilación asistida por radiación de microondas. ${ }^{7}$ Aparicio et al., 2019, realizaron la caracterización química y actividad antimicrobiana del aceite esencial de las hojas de Libanothamnus neriifolius (Asteraceae), que lo obtuvieron mediante el método de hidrodestilación utilizando la trampa de Clevenger8.

La presente investigación, tiene por finalidad realizar el estudio de la composición química y fitoquímico del aceite esencial de la yareta (Smallanthus parviceps (Blake) Rob), con el objetivo de evaluar una posible aplicación en el ámbito farmacéutico.

\section{PARTE EXPERIMENTAL}

Recolección de la muestra. La muestra llamada yareta, fue recolectada el 15 de julio del 2019 por la estudiante de la Escuela de Química de la UNMSM, Marisol Judith Fernández Ruiz, en el distrito de Chumpi, provincia de Parinacochas, departamento de Ayacucho; las coordenadas son: $14^{\circ} 41^{\prime} 38^{\prime \prime} \mathrm{S}, 7^{\circ} 07^{\prime} 27^{\prime \prime} \mathrm{O}$ a una altitud $3207 \mathrm{msnm}$.

La especie Smallanthus parviceps (Blake) Rob. es una planta oriunda del distrito de Chumpi (departamento de Ayacucho) crece cerca del agua de manantial (puquio), por lo cual lo convierten en un lugar apropiado para la evolución y adaptación de la especie vegetal. Esta 
planta es aromática presenta tallos leñosos, hojas ovaladas, flores rojo-naranja en la parte superior, en las figuras 1 y 2, tenemos varias fotos de la especie en estudio. Esta planta es conocida comúnmente como yareta y los lugareños la utilizan como emplastos para inflamación de heridas y golpes.

Identificación de la muestra. La identificación botánica se realizó en el Museo de Historia Natural de la Universidad Nacional Mayor de San Marcos, por el biólogo Hamiltor Wilme Beltrán Santiago, quien nos proporcionó el nombre científico como Smallanthus parviceps (Blake) Rob (constancia N 011-USM-2019). Finalmente, la muestra vegetal entera se llevó al Museo de Historia Natural de la UNMSM y su determinación taxonómica completa, la cual, toda la información está en la tabla 1 .

Extracción del aceite esencial. Se observó que la planta presentaba un aroma particular, bastante similar a un fruto cítrico, por ello se realizó la extracción del aceite esencial mediante el equipo de destilación por arrastre con vapor semi industrial, ver figura 4 . El procedimiento se realizó en el laboratorio de Productos Naturales de la UNMSM de la Facultad de Química e Ingeniería Química. Se agregó $8 \mathrm{~L}$ de agua en el reservorio inferior y en el superior de la cámara extractora, se le agregó 1500 gramos de la planta fresca, la extracción se realizó por 2 horas y se obtuvo al final 1,2 mL de aceite esencial, el cual fue guardado en un vial de color ámbar, limpio, seco y pesado; finalmente se guardó en la refrigeradora.

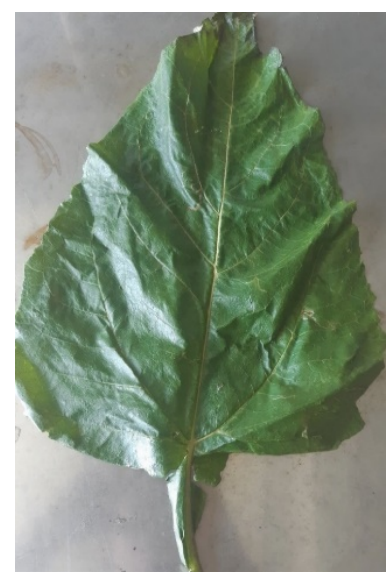

Figura 1. Hoja de la planta Smallanthus parviceps (Blake) Rob.

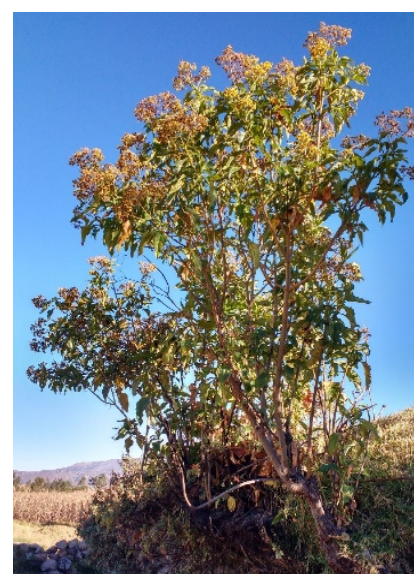

Figura 2. Planta Smallanthus. 
Tabla 1. Taxonomía de la "yareta" Smallanthus parviceps (Blake) Rob.

\begin{tabular}{lc}
\hline CARACTERÍSTICA & CLASIFICACIÓN \\
\hline Reino & Plantae \\
División & Magnoliopsida \\
Clase & Magnoliopsida \\
Subclase & Asteridae \\
Orden & Asterales \\
Familia & Asteraceae \\
Género & Smallantus \\
Especie & Smallantus parviceps (Blake) Rob \\
Nombre común & yareta \\
\hline
\end{tabular}

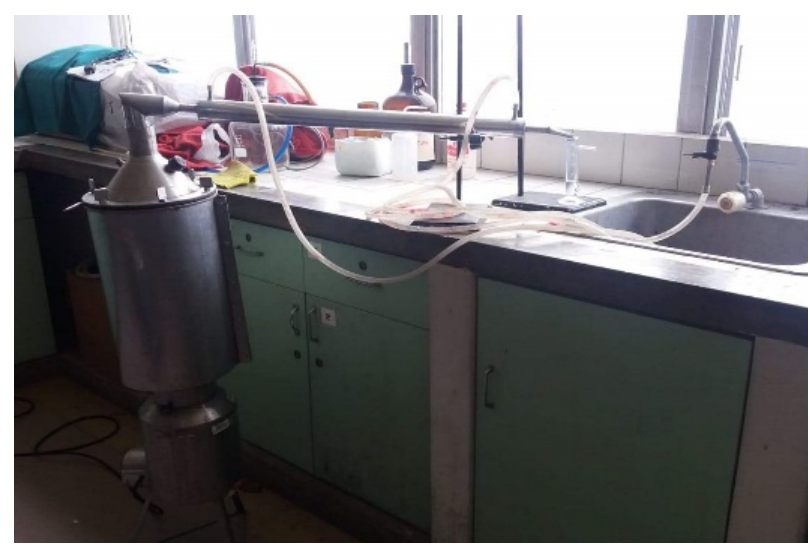

Figura 3. Equipo para extracción de aceites esenciales (Destilador por arrastre con vapor semi industrial)

Cromatografía de gases acoplado a masas. Este análisis se realizó en el laboratorio de cromatografía de la UNSAAC, se empleó un cromatógrafo de Agilent $6890 \mathrm{~N}$, con detector espectrómetro Agilent 5975B, columna Agilent HP-5MS 5 \% Fenil Metil Siloxano, relación de Split 100:1, temperatura incial de $250^{\circ} \mathrm{C}$, flujo de $1 \mathrm{~mL} / \mathrm{min}$.

\section{RESULTADOS Y DISCUSIÓN}

La extracción del aceite esencial de $S$. parviceps (Blake) Rob, fue obtenida a partir de hojas frescas mediante el equipo de destilación por arrastre con vapor semi industrial, el volumen final obtenido fue de 1,2 mL y tiene una coloración amarillo tenue. Presenta un olor que puede ser definido como cítrico, agradable y balsámico, este aroma podría ser de utilidad en industrias de fragancias y productos farmacéuticos. Su rendimiento fue de $0,06 \%$, calculados 
sobre la base del material fresco, este valor es bajo comparado al estudio del aceite esencial de Libanothamnus neriifolius (Asteraceae) que fue de $0,087 \%$, este se realizó mediante una hidrodestilación usando una trampa tipo Clevengery, secando previamente la muestra ${ }^{7}$. La diferencia podría ser debido al método de extracción utilizado y al estado de la muestra, ya que según Ochoa et al. ${ }^{9}$ debería haber un mayor rendimiento usando la destilación por arrastre con vapor, si la muestra está seca. Sin embargo, por el método empleado (destilación por arrastre de vapor) se tiene mayor ventaja de producir menor hidrólisis en relación a la hidrodestilación, la presencia de agua y las altas temperaturas en el método de extracción pueden ocurrir reacciones que favorezcan en la formación de compuestos como alcoholes y ácidos por descomposición de los ésteres causando la disminución en la producción de aceite o formación de derivados. Del análisis fisicoquímico se determinó su densidad relativa 0,75 $\mathrm{g} / \mathrm{mL}\left(25^{\circ} \mathrm{C}\right)$ y su $\mathrm{pH}: 6$.

\section{Composición química del aceite esencial para la especie Smallanthus parviceps (Blake) Rob.}

La identificación de los componentes presentes en el aceite esencial de las hojas de $S$. parviceps (Blake) Rob se realizó comparando los tiempos de retención y los espectros de masas con los datos reportados en la literatura. La cuantificación de los componentes principales se determinó por porcentajes de áreas.

En la tabla 2, se presentan los compuestos identificados por comparación con los índices de retención y con la librería NIST 11; se reportaron que 26 compuestos que representaban más de un $90 \%$ de coincidencia con el espectro de la librería, también se presentan las cantidades relativas que corresponden al porcentaje de abundancia de cada componente dentro del aceite esencial. Se determinó 49 compuestos, entre ellos monoterpenos, diterpenos, sesquiterpenos e hidrocarburos alifáticos, los cuales constituyen cerca de $82,77 \%$ de la composición del aceite esencial. Los monoterpenos representan el 41,87\%, donde el constituyente en mayor abundancia es el $\alpha$-felandreno seguido del p-cimeno y del $\alpha$-pineno.

En cuanto a los diterpenos, estos representan el 5,82 \%, de los cuales el componente mayoritario es el Geranil-p-cimeno, y el Geranil-alfa-terpineno. Los sesquiterpenos encontrados representan el 35,09\%, los componentes mayoritarios encontrados son el cariofileno (sesquiterpeno biciclico), el $\alpha$-curcumeno, $\beta$-curcumeno y al final el óxido de cariofileno (sesquiterpeno biciclico). De los hidrocarburos alifáticos, solo se identificaron 3, estos representan tan solo el 2,96 \% donde el compuesto mayoritario corresponde al tricosano seguido del hexacosano (tabla 3 ). 
Tabla 2. Composición del aceite esencial de hojas de la "yareta.

\begin{tabular}{|c|c|c|c|c|}
\hline Pico & * Compuesto Fitoquímico & Familia & $\begin{array}{l}* \mathrm{~T}_{\mathrm{r}} \\
(\mathrm{min})\end{array}$ & \% Área \\
\hline 1 & $\alpha$-pineno & M & 6,10 & 3,66 \\
\hline 2 & 4-metilen- 1-(1-metiletil)-biciclo [3.1.0] hexano & M & 7,16 & 2,74 \\
\hline 3 & $\beta$-mirceno & M & 7,65 & 1,03 \\
\hline 4 & $\alpha$-felandreno & M & 8,09 & 18,28 \\
\hline 5 & p-cimeno & M & 8,68 & 8,11 \\
\hline 6 & D-Limoneno & M & 8,79 & 0,42 \\
\hline 7 & $\gamma$-terpineno & M & 9,73 & 0,60 \\
\hline 8 & 2-metil-4-bromo-1-buteno & - & 11,16 & 0,35 \\
\hline 9 & Terpinen-4-ol & M & 13,66 & 0,97 \\
\hline 10 & 2-metoxi-4-metil-1-(1-metiletil)-benceno & M & 15,58 & 0,91 \\
\hline 11 & 5-hidroximetilfurfural & - & 16,17 & 0,68 \\
\hline 12 & (R)- Acetato de lavandulilo & M & 17,46 & 1,23 \\
\hline 13 & 2-acetilciclopentanona & - & 18,31 & 1,23 \\
\hline 14 & 1-butil-2-ciclohexen-1-ol & - & 18,93 & 0,54 \\
\hline 15 & (-)-Aristoleno & $\mathrm{S}$ & 19,15 & 0,40 \\
\hline 16 & Alfa-copaeno & $\mathrm{S}$ & 20,20 & 0,49 \\
\hline 17 & 3,5-dimetil-ciclohexanol & - & 20,77 & 0,79 \\
\hline 18 & 3-etoxi-5-metil-1H-pirazol & - & 21,49 & 0,34 \\
\hline 19 & Cariofileno & $\mathrm{S}$ & 21,61 & 8,73 \\
\hline 20 & 1- metil-4-(1-metiletil)- 1,3-ciclohexadieno & - & 21,79 & 0,74 \\
\hline 21 & Humeleno & $\mathrm{S}$ & 22,63 & 1,92 \\
\hline 22 & Germacreno D & $\mathrm{S}$ & 23,47 & 1,86 \\
\hline 23 & Ácido 2-bromopropiónico, éster 2-feniletílico & - & 23,63 & 0,65 \\
\hline 24 & $\beta$-Gurjuneno & S & 23,88 & 1,20 \\
\hline 25 & Neryl (S)-2-metilbutanoato & - & 24,31 & 1,83 \\
\hline 26 & $\alpha$-terpineno & M & 24,42 & 0,22 \\
\hline 27 & d-Cadineno & - & 24,74 & 1,77 \\
\hline 28 & (-)- $\alpha$-Gurjuneno & - & 25,15 & 0,37 \\
\hline 29 & Aloaromadendreno & - & 25,71 & 0,53 \\
\hline 30 & Nerolidol & S & 25,91 & 2,92 \\
\hline 31 & Espatulenol & S & 26,34 & 1,32 \\
\hline 32 & Oxido de cariofileno & $\mathrm{S}$ & 26,50 & 3,53 \\
\hline 33 & Epóxido de humeleno II & - & 27,23 & 0,73 \\
\hline 34 & 4-fenil-2-pirrolidinona & - & 28,11 & 0,75 \\
\hline 35 & p-cimeno & M & 34,68 & 0,51 \\
\hline 36 & Trans-(2-clorovinil) trimetilsilano & - & 35,11 & 0,51 \\
\hline 37 & Geranil- $\alpha$-terpineno & $\mathrm{D}$ & 36,13 & 2,27 \\
\hline 38 & Geranil-p-cimeno & $\mathrm{D}$ & 36,19 & 1,31 \\
\hline 39 & $\beta$-curcumeno & $\mathrm{S}$ & 37,54 & 7,27 \\
\hline 40 & $\alpha$-curcumeno & $\mathrm{S}$ & 37,59 & 5,45 \\
\hline 41 & 1,5,9,13-tetradecatetraeno & HA & 38,20 & 0,78 \\
\hline 42 & Ácido 2-propenoico, 3-fenil-,2-feniletil éster & - & 38,63 & 0,64 \\
\hline 43 & $\begin{array}{l}\text { biciclo [3.1.1] heptano, 6-metil-2-metilen-6- (4-metil-3-pentenilo) -, } \\
{[1 \mathrm{R}-(1 \alpha, 5 \alpha, 6 \beta)]}\end{array}$ & M & 39,18 & 0,69 \\
\hline 44 & Geranil-p-cimeno & $\mathrm{D}$ & 39,25 & 0,56 \\
\hline 45 & 5 H-5-Metil-6,7- dihidrociclopentapirazina & M & 39,92 & 2,50 \\
\hline 46 & Geranil-p-cimeno & $\mathrm{D}$ & 39,97 & 1,68 \\
\hline 47 & 2- metilbenzil cianuro & - & 41,31 & 1,84 \\
\hline 48 & Tricosano & HA & 43,83 & 1,34 \\
\hline 49 & Hexacosano & HA & 47,81 & 0,84 \\
\hline
\end{tabular}


Además, se sabe que $\alpha$-pineno constituye el 3,66 \% del aceite esencial y el $\alpha$-felanfreno el $18,28 \%$, el cual es muy bajo comparado con el estudio de la Libanothamus neriifolius (B. ex H) Ernst (Asteraceae), ya que contiene de $\alpha$-pineno 13,57 \% y de $\alpha$-felanfreno 19,86\% 7. Del estudio de comparación se reportaron otros compuestos mayoritarios como el $\beta$-felandreno $(29,04 \%)$ y $\alpha$-tujeno $(12,35 \%)$ mientras que en nuestra investigación fueron identificados al $\beta$ -curcumeno $(7,11 \%$ ) y al cariofileno $(8,73 \%)$ como compuestos mayoritarios, lo cual indica la variación en la composición química de metabolitos volátiles de esta especie (Asteraceae).

Tabla 3. Clasificación de los compuestos identificados por GC-MS en el aceite esencial de la "yareta"

\begin{tabular}{lc}
\hline \multicolumn{1}{c}{ Clasificación } & Porcentaje (\%) \\
\hline Monoterpenos (M) & 41,87 \\
Diterpenos (D) & 5,82 \\
Sesquiterpenos (S) & 35,09 \\
Hidrocarburos Alifáticos (HA) & 2,96 \\
Otros & 14,29 \\
\hline
\end{tabular}

La diferencia se podría atribuir a las condiciones físicas (clima, temperatura, variantes fisiológicas, etc.) que contribuyeron con la evolución de esta especie para adaptarse y sobrevivir en el medio que le rodea según Figueiredo ${ }^{10}$. Por otra parte, según la investigación realizada por Gobbo-Neto y Lopez ${ }^{11}$, en las plantas medicinales existen diferentes factores que influyen en el contenido de los metabolitos secundarios: estado, temperatura, radiación UV, nutrientes del suelo, altitud, composición atmosférica, entre otros.

Sin embargo, podemos resaltar que el aceite esencial de la $S$. parviceps (Blake) Rob presenta en mayor proporción al p-cimeno $(11,81 \%)$ y al terpinen-4-ol $(0,97 \%)$ comparado con el estudio de Wedelia calycina L.C. Rich. (Asteraceae) que contiene de p-cimeno 0,05\% y de terpinen-4-ol 0,95\% 12. También podemos indicar que de los 49 componentes encontrados en el cromatograma, se resaltan 6 de ellos por su abundancia, tenemos al $\alpha$-felandreno (Rt:8,094, A\%:18,28), al o-cimeno (Rt:8,680, A\%:8,11), al cariofileno (Rt:21,614, A\%:8,73), a los isómeros $\alpha$-curcumeno (Rt:37,588, A\%:5,45) y al $\beta$-curcumeno (Rt:37,537, A\%:7,27), al $\alpha$-pineno (Rt:6,098, A\%:3,66) y al óxido de cariofileno (Rt:7,160, A\%:3,66), figura 4. De los 7 componentes abundantes podemos indicar que el $\alpha$-felandreno tiene potenciales beneficios para combatir el cáncer (estudio in vitro) y presenta propiedades antiinflamatorias (estudio en roedores). A este compuesto se le atribuyen numerosas propiedades, dentro de las cuales tenemos, antinflamatorias, antinconceptivas y actividad antiproliferativa según Siqueira et $a l^{12}$. 

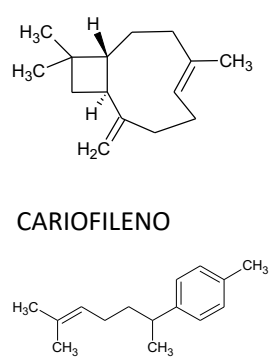

B-CURCUMENO<smiles>Cc1ccc(C(C)C)cc1</smiles>

p-CIMENO

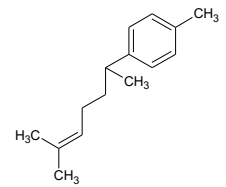

$\alpha$-CURCUMENO<smiles>CC1=CCC(C(C)C)C=C1</smiles>

$\alpha$-FELANDRENO

ÓXIDO DE CARIOFILENO<smiles>CC1=CC[C@H]2CC1C2(C)C</smiles>

$\alpha$-PINENO

Figura 4. Estructuras de los compuestos mayotitarios del AE presentes en hojas de la planta Smallanthus parviceps (Blake) Rob.

En cambio, el carofileno solo tiene propiedades antiinflamatorias; por otro lado, el p-cimeno posee propiedades insecticidas y medicinales ${ }^{13}$ y la presencia de $\alpha$-pineno en el aceite esencial, muestra un papel potencial para el tratamiento del dolor inflamatorio y neuropático ${ }^{14}$

\section{CONCLUSIONES}

De los resultados del análisis por GC-MS del aceite esencial de la planta Smallanthus parviceps (Blake) Rob se puede concluir que se identificaron 49 compuestos, de estos hay dos que son mayoritarios, el $\alpha$-felandreno y el carofileno, los cuales son monoterpeno hidrocarburado que presenta propiedades antiinflamatorias, estos compuestos pueden ser aplicados en el ámbito farmacéutico.

El presente estudio es un aporte a las investigaciones fitoquímicas de la familia Asteraceae en el Perú y en particular del género Smallanthus, ya que este es el primer reporte a nivel nacional en cuanto a la constitución de metabolitos volátiles de un género que cuenta con escasos estudios químicos.

\section{AGRADECIMIENTO}

Agradecemos al responsable del Laboratorio de Productos Naturales, la Prof. Mg. Gloria Tomas Chota, por permitirnos trabajar y hacer la extracción del aceite esencial. También un agradecimiento por los análisis fisicoquímicos y otros análisis al Dr. Julio Santiago Contreras, responsable del LIDQO (Laboratorio de Investigación y Desarrollo en Química Orgánica). 


\section{REFERENCIAS BIBLIOGRÁFICAS}

1. Li Y, Fabiano S. Essential Oils as Reagents in Green Chemistry. New York: Springer International Publishing; 2014.

2. Kahriman N, Tosun G, Terzioglu S, Karaoglu S, Yayh N. Chemical Composition and Antimicrobial Activity of the Essential Oils from the Flower, Leaf, and Stem of Senecio pandurifolius. Rec Nat Prod. 2011; 5(2): 82 - 91

3. Leyva M, Tacoronte JE, Marquetti MC, Tiomno O, Montada D, Mesa A. Actividad insecticida de aceites esenciales de plantas en larvas de Aedes aegypti (Diptera: Culicidae). Rev Cubana Med Trop. 2007; 60 (1): 78-82

4. Vitali M. Anatomía foliar del género Smallanthus (Asteraceae, Milleriaea). Bol Soc Argentina Bot. 2017; 52(3): 463-472.

5. Guzmán A, Barrera D. Estudio fitoquímico de hojas y flores de Smallanthus pyramidales (Triana) H. Bob (ARBOLOCO) y su uso en la recuperación de humedales de Bogotá. Colombia Forestal. 2011; 14(1), 41-50.

6. Arnao I, Suárez S, Cisneros R, Trabuco J. Evaluación de la capacidad antioxidante de los extractos acuosos de la raíz y las hojas de Smallanthus sonchifolius (yacón). Rev Soc Quim Perú. 2012; 78(2): 120-127.

7. Mendoza D, Parra L, Loza S. Capacidad captadora de radicales libres del aceite esencial y extractos etanólicos de yacón (Smallanthus sonchifolius Poepp. \& endl) H. Robinson, cultivado en Colombia. Revista Biosalud. 2014; 13(2): 9-23.

8. Aparicio R, Rojas L, Velasco J, Usubillaga A, Sosa M, Rojas J. Caracterización química y actividad antimicrobiana del aceite esencial de las hojas de Libanothamnus neriifolius (Asteraceae). Rev Perú Biol. 2019; 26(1): 96 - 100.

9. Ochoa K, Paredes L, Bejarano D, Silva R. Extracción, caracterización y evaluación de la actividad antibacteriana del aceite esencial de Senecio graveolens Wedd. Scientia Aagropecuaria. 2012; (3): 291-302.

10. Gobbo-Neto L, Lopes NP. Plantas medicinales: factores influyendo en el contenido de metabolitos secundarios. Quím Nova; 2007; 30 (2): 274-381.

11. Corella R, Ortega M. Importancia del aceite esencial y la producción de orégano Lippia palmeri Watson en el estado de Sonora. Biotecnia. 2013; (1): 57-64.

12. Siqueira HDAS, Neto BS, Sousa DP, Gomes BS, da Silva FV, Cunha F, et al. El $\alpha$ -felandreno, un monoterpeno cíclico, atenúa la respuesta inflamatoria mediante la inhibición de la migración de neutrófilos y la desgranulación de mastocitos. Ciencias de la vida. 2016, 160: 27-33.

13. Bruzual H, Guzmán W, Crescente O, Lanza J. Aceite esencial de Wedelia calycina (Asteraceae): composición química, actividad antibacteriana y antifúngica. Saber, Universidad de Oriente, Venezuela. 2015; (1): 87-93

14. Quintão NL, da Silva GF, Antonialli CS, Rocha LW, Cechinel FV, Ciccio JF Composición química y evaluación del efecto anti-hipernociceptivo del aceite esencial extraído de las hojas de Ugni myricoides en modelos inflamatorios y neuropáticos de dolor en ratones. Planta Med. 2010; 76: 1411-1418. 